Cahiers de la recherche sur les droits

Cahiers

Fur les Droits fondamentaux

5 | 2006

L'enfant

\title{
Les modes de participation de l'enfant aux procédures judiciaires
}

\section{Adeline Gouttenoire}

\section{(2) OpenEdition}

12 Journals

Édition électronique

URL : https://journals.openedition.org/crdf/7167

DOI : $10.4000 /$ crdf.7167

ISSN : 2264-1246

Éditeur

Presses universitaires de Caen

Édition imprimée

Date de publication : 31 décembre 2006

Pagination : 59-64

ISBN : 978-2-84133-277-9

ISSN : $1634-8842$

\section{Référence électronique}

Adeline Gouttenoire, "Les modes de participation de l'enfant aux procédures judiciaires », Cahiers de la recherche sur les droits fondamentaux [En ligne], 5 | 2006, mis en ligne le 15 décembre 2020, consulté le 14 novembre 2022. URL : http://journals.openedition.org/crdf/7167 ; DOI : https://doi.org/10.4000/ crdf.7167 


\title{
Les modes de participation de l'enfant aux procédures judiciaires
}

\author{
Adeline GOUTTENOIRE \\ Professeur \\ Faculté de droit de l'Université Montesquieu - Bordeaux IV
}

\author{
I. L'audition du mineur \\ II. Le droit de veto \\ III. Le consentement du mineur \\ IV. La représentation ad hoc des intérêts de l'enfant \\ V. L'action personnelle de l'enfant
}

L'évolution contemporaine des droits de l'enfant notamment sur le plan international et européen a mis en lumière l'idée que l'enfant ne peut subir les décisions des adultes le concernant, fussent-ils ses parents ou un juge, sans être au moins consulté. Les différentes sources contemporaines des droits de l'enfant consacrent ce qu'on peut qualifier de droit de l'enfant à participer aux décisions qui le concernent.

La Convention internationale des droits de l'enfant, désormais d'applicabilité directe en France même aux yeux de la Cour de cassation ${ }^{1}$, au moins pour ses dispositions self executing, contient dans son article 12 le droit de l'enfant d'exprimer librement son opinion sur toute question l'intéressant, ses opinions étant dûment prises en considération eu égard à son âge et à son degré de maturité. L'alinéa 2 de cet article dispose qu'à cette fin, on donnera notamment à l'enfant la possibilité être entendu dans toute procédure judiciaire ou administrative l'intéressant, soit directement, soit par l'intermédiaire d'un représentant ou d'un organisme approprié, de façon compatible avec les règles de procédure de la législation nationale.

La Convention de La Haye du 25 octobre 1980 relative aux enlèvements d'enfant organise également la participation de l'enfant au processus judiciaire en faisant du refus de ce dernier de retourner dans son pays d'origine un motif pour le juge de ne pas ordonner son retour, qui est de principe après un enlèvement illicite ${ }^{2}$.

Le droit européen a également fait de la participation de l'enfant aux décisions qui le concernent, particulièrement lorsqu'elles s'inscrivent dans le cadre judiciaire, un élément non négligeable du processus décisionnel. De manière générale, la Cour européenne des droits de l'homme accorde en effet à la parole de l'enfant un poids certain, sous réserve que celle-ci soit exprimée dans des conditions sereines ${ }^{3}$. Plus précisément, les dispositions conventionnelles européennes concernant les droits de

1. Civ. $1^{\text {re }}, 18$ mai 2005, Dr. fam., 2005, comm. ${ }^{\circ}$ 156, obs. A. Gouttenoire; F. Boulanger, "Applicabilité directe de la CIDE et intérêt supérieur de l'enfant ", D., 2006, p. 554 .

2. A. Gouttenoire, «La parole de l'enfant enlevé », in Les Enlèvements d'enfants à travers les frontières, H. Fulchiron (dir.), Bruxelles, Bruylant, 2004, p. 349.

3. CEDH, Sophia Gudrun Hansen c. Turquie, 23 septembre 2003. 
l'enfant insistent sur la nécessité d'associer l'enfant aux débats dont il est l'objet. Il en va ainsi de la Convention européenne sur l'exercice des droits de l'enfant de 1996, émanant du Conseil de l'Europe et entrée en vigueur le $1^{\text {er }}$ juillet 2000 , de même que de la plus récente Convention sur les relations personnelles concernant les enfants du 15 mai 2003. La Charte des droits fondamentaux de l'Union européenne n'est pas en reste puisque dans son article 24 elle consacre, de manière générale, la possibilité pour les enfants d'exprimer librement leur opinion ${ }^{4}$.

La participation de l'enfant aux décisions qui le concernent a également trouvé sa place dans le droit interne notamment dans les textes les plus récents. L'article 371 du Code civil dispose depuis la loi du 4 mars 2002 que les parents doivent associer l'enfant aux décisions le concernant selon son âge et son degré de maturité. La difficulté consiste à savoir quelle sanction cette obligation pourrait recevoir : l'éventuel conflit entre les parents et l'enfant ne relève semble-t-il pas de la compétence du juge aux affaires familiales et il peut relever du juge des enfants seulement s'il place ce dernier dans une situation de danger.

Peuvent être évidemment rangées dans la catégorie des règles qui permettent la participation de l'enfant aux décisions qui le concernent les différentes dispositions qui prévoient son audition ou / et son consentement. Celles-ci ne sont pas cantonnées au droit processuel; le droit médical impose en principe la recherche du consentement du patient mineur et lui accorde même dans certains cas un réel pouvoir de décision, négatif, à travers un droit de veto, ou positif, à travers la reconnaissance d'une capacité spéciale de consentir à l'acte ${ }^{5}$. Il faut enfin évoquer les textes relatifs à l'administrateur $a d h o c^{6}$ qui en matière judiciaire ou extrajudiciaire permet à l'enfant d'être présent dans les débats par l'intermédiaire d'un représentant spécial chargé de défendre spécifiquement ses intérêts lorsqu'ils sont en opposition avec ceux de ses parents ${ }^{7}$.

Les manifestations du droit de l'enfant de participer aux décisions qui le concernent se révèlent ainsi diversifiées dans leur source comme dans leurs modalités. Cette étude vise à opérer une présentation générale des différentes formes de participation de l'enfant aux procédures judiciaires, dans un ordre croissant en fonction de l'intensité de cette participation à la procédure qu'elles permettent.

\section{L'audition du mineur}

L'audition du mineur constitue la forme minimaliste de la participation de l'enfant à la procédure qui le concerne. Elle s'inscrit en principe dans le cadre de procédures dans lesquelles le mineur n'est pas partie, ses droits subjectifs n'étant pas mis en cause dans la procédure. L'audition de l'enfant présente un intérêt seulement lors- que la décision se fonde non pas sur des éléments objectifs tels que la vérité biologique dans le cadre des actions relatives à la filiation, mais sur des éléments plus subjectifs tels que l'intérêt de l'enfant. Elle a pour objet de permettre à l'enfant de donner son avis et non pas de le faire témoigner de manière objective. Le but est de lui permettre d'exprimer ses sentiments et non pas de connaître par sa voix la réalité de la situation familiale.

L'audition s'inscrit essentiellement dans le cadre des procédures relatives à l'autorité parentale, qui concernent le mineur en fait mais pas en droit. Si ses droits subjectifs ne sont pas mis en œuvre, l'issue de la procédure a en effet un impact évident sur son mode de vie. Privé d'intérêt pour agir, le mineur peut cependant exprimer un avis sur sa propre situation, laquelle est l'objet de la procédure. Prévue par la plupart des conventions relatives aux droits de l'enfant (Convention internationale des droits de l'enfant, Convention du Conseil de l'Europe sur l'exercice des droits de l'enfant mais aussi Règlement Bruxelles II bis) et par l'article 388-1 du Code civil, l'audition est expressément ou implicitement subordonnée au discernement de l'enfant, sans que ne soit jamais prévue la manière dont ce discernement doit être mesuré.

L'audition de l'enfant en justice n'a été rendue systématique par aucun texte; toutefois son caractère obligatoire pour le juge, lorsqu'elle est sollicitée par l'enfant, varie selon les sources : l'article 388-1 du Code civil laisse au juge le pouvoir de refuser d'entendre l'enfant, à condition qu'il motive sa décision, laquelle ne peut cependant faire l'objet d'aucun recours. La Cour de cassation a d'ores et déjà affirmé dans l'arrêt du 18 mai $2005^{8}$ que la demande d'audition de l'enfant ne doit pas rester sans réponse. En déclarant l'article 12 de la CIDE d'applicabilité directe, la Haute Cour permet au justiciable de fonder sa demande d'audition sur ce texte plus favorable à l'audition que ne l'est l'article 388-1 du Code civil. Les exigences de la Convention internationale en matière d'audition de l'enfant sont en effet plus grandes que celles du droit français puisque l'article 12-2 impose aux États de donner à l'enfant la possibilité d'être entendu dans toute procédure le concernant, selon des modalités prévues par la législation nationale. L'enfant est ainsi titulaire, au regard des dispositions de la CIDE, d'un véritable droit d'être entendu lorsqu'il le souhaite - comme l'affirme d'ailleurs la Cour de cassation dans l'arrêt du 18 mai 2005 - alors que le texte français ne lui accorde que le droit de solliciter son audition, qui peut être refusée par le juge. L'article 388-1 pourrait ainsi être supplanté par l'article 12-2 de la Convention internationale désormais susceptible d'être invoqué par un enfant à l'encontre du refus du juge d'accéder à sa demande d'audition. Le projet de réforme de la protection de l'enfance déposé à l'Assemblée nationale le 3 mai 2006 par le ministre de la Santé et de la Solidarité, par souci de

\footnotetext{
4. Commentaire de la Charte des droits fondamentaux de l'Union européenne, F. Picot, F. Burgorgue-Larsen (dir.), Bruxelles, Bruylant, 2005, article 24 relatif aux droits de l'enfant p. 332.

5. D. et M. Duval Arnould, Droit et santé de l'enfant, Paris, Masson, 2002.

6. Art. 388-2 et 389-3 C. civ.

7. A. Gouttenoire, Rep. Dalloz proc. civ., $\mathrm{v}^{\circ}$ Mineurs, $\mathrm{n}^{\circ} 160 \mathrm{sq}$.

8. Civ. $17^{\text {re }}, 18$ mai 2005.
} 
mettre le droit français en conformité avec le droit international et européen, prévoit cependant de consacrer un véritable droit de l'enfant d'être entendu lorsqu'il le demande en supprimant le pouvoir du juge de refuser l'audition dans cette hypothèse.

Le règlement communautaire du 27 novembre 2003 $n^{0} 2201 / 2003$ relatif à la compétence, la reconnaissance et l'exécution des décisions en matière matrimoniale ${ }^{9}$ va également plus loin que le droit interne dans la reconnaissance d'un droit de l'enfant d'être entendu dans les procédures judiciaires le concernant. Il subordonne en effet la force exécutoire dans les pays de l'Union d'une décision rendue dans un État membre à la possibilité qui a été donnée à l'enfant d'être entendu au cours de la procédure « à moins qu'une audition n'ait été jugée inappropriée eu égard à son âge ou à son degré de maturité ${ }^{10}$. Ce dispositif, fruit d'un compromis entre les pays de l'Union européenne qui souhaitaient subordonner la force exécutoire de la décision à l'audition du mineur et ceux qui considéraient qu'une telle condition était excessive, s'il exclut l'audition automatique du mineur, lui accorde indirectement mais incontestablement le droit d'être entendu s'il le souhaite et impose même sans doute aux autorités compétentes de proposer à l'enfant d'être entendu. Il est remarquable, parce qu'exceptionnel, que le mécanisme soit assorti d'une sanction, la décision prise sans que l'enfant n'ait eu la possibilité d'être entendu étant en effet susceptible de ne pas être reconnue ${ }^{11}$.

Certains textes internes, plus spécifiques, imposent au juge d'entendre l'enfant avant de rendre sa décision. Il en va ainsi dans le cadre de l'assistance éducative ${ }^{12}$; par ailleurs, selon l'article 477 du Code civil le juge doit entendre le mineur avant de l'émanciper. Même s'il ne s'agit pas d'une procédure judiciaire, il n'est pas sans intérêt de préciser que la loi n ${ }^{\circ} 98-381$ a inséré, dans l'article 411 du Code civil, l'obligation pour le juge des tutelles d'entendre préalablement à la réunion du Conseil de famille le mineur capable de discernement dans les conditions prévues à l'article 388-1.

\section{Le droit de veto}

Il faut entendre par droit de veto le mécanisme selon lequel la décision est subordonnée à l'absence de refus de l'enfant; dans une telle hypothèse, l'opposition de l'enfant interdit que la décision soit prise; elle peut en revanche être rendue si l'enfant acquiesce ou n'exprime rien. La Convention de La Haye du 25 octobre 1980, relative aux enlèvements internationaux d'enfant, accorde ainsi à celuici un véritable droit de veto en permettant à l'autorité administrative ou judiciaire compétente pour ordonner son retour de ne pas prendre cette décision lorsqu'il refuse de retourner dans son pays d'origine ${ }^{13}$. La Cour de cassation semble cependant faire une interprétation restrictive de cette disposition en considérant que la seule opposition de l'enfant ne peut justifier le rejet de la demande de retour ${ }^{14}$, semblant opérer un contrôle sur les motivations avancées par l'enfant pour justifier son refus de retourner dans le pays de sa résidence habituelle.

Un tel droit de veto est également accordé à l'enfant en droit français dans le cadre d'un prélèvement de moelle osseuse $^{15}$. Ce mécanisme ne conduit pas à imposer l'audition de l'enfant, ni à suivre son avis lorsqu'il est positif, mais seulement à ne pas lui imposer une décision qu'il rejette.

\section{Le consentement du mineur}

Forme plus intense de la participation de l'enfant au processus de décision, le consentement fait de l'enfant un acteur à part entière de la procédure puisque la décision ne peut être prise sans qu'il y adhère formellement. Plus encore qu'une audition, qu'il implique cependant, le consentement du mineur garantit sa participation à la procédure. Cette exigence de consentement doit se distinguer de la recherche de l'adhésion du mineur à la décision envisagée, présente dans le cadre de l'assistance éducative ou encore en matière médicale où le consentement de l'enfant doit être recherché mais pas exigé ${ }^{16}$. Le consentement de l'enfant est rarement exigé dans le cadre des procédures judiciaires ; il ne concerne en réalité que des hypothèses ponctuelles: le changement de nom et de prénom ${ }^{17}$, l'adoption $^{18}$, et uniquement lorsqu'il s'agit d'un enfant de plus de 13 ans. Le consentement de l'enfant s'inscrit dans le cadre de procédures dans lesquelles l'enfant n'a ni qualité ni capacité pour agir. Qu'il s'agisse du nom ou de l'adoption, les procédures dans lesquelles le consentement du mineur

9. Dit Bruxelles II bis; A. Bigot, «Le nouveau règlement communautaire du 27 novembre 2003 en matière matrimoniale et de responsabilité parentale », Dr. fam., 2004, Chron. $\mathrm{n}^{\circ} 8$.

10. H. Gaudement-Tallon, «La compétence, la reconnaissance et l'exécution des décisions concernant les enfants au sein de l’Union européenne », in La Condition juridique du mineur. Aspects internes et internationaux. Questions d'actualité, J.-J. Lemouland (dir.), Paris, Litec, 2004. Le règlement 1347/ 2000 dit «Bruxelles II » prévoyait déjà qu’un État pouvait refuser de reconnaître une décision concernant un enfant lorsque celui-ci n’avait pas pu être entendu alors qu'il aurait dû l'être compte tenu de son âge et de sa maturité.

11. A. Gouttenoire, «L'audition de l'enfant dans le règlement de Bruxelles II bis », in Le Nouveau Droit communautaire du divorce et de la responsabilité parentale, H. Fulchiron et C. Nourrissat (dir.), Paris, Dalloz (Actes), 2005, p. 201.

12. Art. 1182 NCPC.

13. A. Gouttenoire, «La parole de l'enfant enlevé», p. 348 sq.

14. Civ. $1^{\text {re }}, 14$ février 2006, D., 2006, p. 672 ; la cour d'appel retient que les éléments de fait invoqués par les enfants pour s'opposer à leur retour au Canada, lieu de résidence de leur père, ne sont manifestement plus actuels et ne peuvent caractériser un danger futur de nature à les placer dans une situation intolérable lorsqu'ils sont sous la responsabilité paternelle.

15. Art. 1231-3 al. 5 C. santé publique « Le refus du mineur fait obstacle au prélèvement ».

16. D. et M. Duval-Arnould, Droit et santé de l'enfant, $\mathrm{n}^{\circ} 28$.

17. Art. $61-3$ et 60 al. 2 C. civ.

18. Art. 345 al. 3 C. civ. 
est exigé mettent en effet en œuvre des droits parentaux et non des droits subjectifs de l'enfant. Toutefois, à la différence de l'audition dont l'objectif est de connaître l'avis de l'enfant sur la réalité factuelle de sa situation, le consentement implique une prise de position à propos d'une décision relative à sa situation juridique, l'adoption comme le changement de nom modifiant son état civil.

\section{La représentation ad hoc des intérêts de l'enfant}

L'administrateur ad hoc, chargé de représenter les intérêts de l'enfant dans toute procédure le concernant, intervient dans le cadre de la procédure civile lorsque les intérêts de ce dernier sont en opposition avec ceux de ses représentants légaux ${ }^{19}$ et dans le cadre de la procédure pénale ${ }^{20}$ lorsque la protection de l'enfant que devraient assurer ses parents est insuffisante. L'administrateur ad hoc est en principe chargé de représenter l'enfant dans les procédures dans lesquelles celui-ci est partie, et a donc intérêt et qualité pour agir, mais sans avoir la capacité d'agir lui-même : c'est le cas du mineur victime d'une infraction pénale, c'est le cas également sans doute du mineur sans discernement objet d'une procédure d'assistance éducative que le décret du 15 mars 2002 a rendu incapable d'exercer lui-même ses droits procéduraux ${ }^{21}$.

La Cour de cassation a plus largement admis que l'administrateur ad hoc puisse intervenir pour représenter l'enfant concerné par une procédure relative à l'autorité parentale $^{22}$, même ce dernier n'est pas partie à cette procédure. Si cette jurisprudence va dans le sens d'une plus grande association du mineur aux procédures relatives à l'autorité parentale, elle suscite des interrogations quant aux pouvoirs et au rôle de l'administrateur ad hoc dans de telles circonstances. Depuis l'entrée en vigueur de la loi du 4 mars 2002 relative à l'autorité parentale, l'administrateur ad hoc intervient également de manière systématique aux côtés de l'enfant étranger isolée ${ }^{23}$; la deuxième hypothèse de désignation automatique d'un administrateur ad hoc pour l'enfant a été supprimée avec l'action en désaveu par la réforme de la filiation du 4 juillet $2005^{24}$.

On peut se demander si le recours à l'administrateur ad hoc pourrait permettre au mineur de prendre l'initiative d'intenter lui-même une procédure pour laquelle il a intérêt pour agir sans en avoir la capacité, par exemple une action en responsabilité ou une action en recherche ou en contestation de filiation ; le mineur pourrait-il saisir le juge des tutelles sur le fondement de l'article 3882 pour lui demander de désigner un administrateur ad hoc, en arguant d'une opposition d'intérêt entre lui et ses représentants légaux, notamment parce que ceux-ci n'ont pas intérêt à ce que l'action soit intentée, par exemple parce qu'elle est intentée contre l'un d'entre eux ou un de leurs proches? L'administrateur ad hoc serait chargé, le cas échéant, par le juge des tutelles d'intenter l'action au nom de l'enfant. Aucune décision ne permet en l'état du droit positif de répondre à cette question. La réponse est délicate parce qu'au-delà de l'incapacité du mineur, l'initiative de celui-ci se heurte à l'autorité parentale, la désignation d'un administrateur ad hoc constituant une atteinte à celle-ci. Il n'est pas certain que le juge des tutelles admettre une telle désignation, l'intervention de l'administrateur ad hoc ayant été plutôt envisagée par le législateur pour permettre à l'enfant de participer à une procédure dont l'initiative a été prise par quelqu'un d'autre que lui.

L'intervention d'un représentant spécial du mineur, dont ce dernier peut solliciter lui-même la désignation, constitue tout de même un outil essentiel de l'autonomie procédurale du mineur et une des meilleures garanties de l'exercice de ses droits, alliant participation de l'enfant et protection. Il reste que le mineur est tenu de passer par un intermédiaire ce qui, parvenu à un certain degré de maturité, peut paraître inadapté.

\section{L'action personnelle de l'enfant}

La possibilité pour l'enfant d'agir lui-même en justice est évidemment le mode de participation au processus judiciaire le plus complet. Il nécessite que l'enfant réunisse toutes les conditions de l'action en justice : intérêt, qualité et capacité pour agir. L'incapacité de principe du mineur dont l'objectif est de protéger l'enfant contre luimême et contre ceux qui pourraient profiter de sa crédulité est logiquement écartée lorsque les nécessités de la protection qui la fonde n'existent plus, soit que ses droits fondamentaux aient été atteints - il peut alors saisir la Cour européenne des droits de l'homme - soit que confronté au danger il participe à sa protection en saisissant le juge des enfants ou en exerçant des droits procéduraux dans le cadre de la procédure d'assistance éducative, s'il est doué de discernement ${ }^{25}$.

Il faut ajouter à ces îlots de capacité l'hypothèse du mineur parent, du mineur futur époux, du mineur salarié

19. Art. 388-2 C. civ.

20. Art. 706-50 C. procédure pénale; la Cour de cassation a récemment admis cependant qu'un juge des tutelles puisse désigner sur le fondement de l'article 388-2 du Code Civil un administrateur ad hoc chargé de représenter un enfant dans le cadre d'une procédure pénale : civ. $17^{\text {re }}, 25$ octobre 2005 , Dr. fam., 2006, comm. $\mathrm{n}^{\circ}$ 77, obs. A. Gouttenoire.

21. A. Gouttenoire, «La réforme imparfaite de l'assistance éducative », Dr. fam., 2002, Étude ${ }^{\circ} 14$.

22. Civ. $1^{\text {re }}, 23$ février 1999: Bull. civ. I, $n^{\circ} 66$; Dr. fam., 1999, comm. ${ }^{\circ}$ 146, obs. A. Gouttenoire-Cornut, pour une procédure de délégation de l'autorité parentale; dans le même sens, Nîmes, 10 août 1993 : Jurisdata 030583.

23. A. Gouttenoire, «La protection de l'enfant par la loi du 4 mars 2002 », Dr. fam., 2002, chron. $n^{\circ} 27$.

24. Ordonnance $\mathrm{n}^{\circ}$ 2005-759 du 4 juillet 2005, JO 6 juillet 2005 p. 11159; J. Hauser, «Des filiations à la filiation», RJPF, 2005-9 / 9, p. 6 ; P. Murat, « La filiation simplifiée », Dr. fam., 2005, Focus p. 4; T. Garé, «La réforme de la filiation », JCP, 2005, Act. p. 1491 ; F. Dekeuwer-Defossez, «Le nouveau droit de la filiation : pas si simple ! ", Lamy Droit civil, $\mathrm{n}^{\circ}$ 21, p. 2 ; «Dossier spécial filiation », Dr. fam., $2006, \mathrm{n}^{\circ} 1$.

25. A. Gouttenoire, Rep. Proc. civ., $\mathrm{v}^{\circ}$ Mineurs, $\mathrm{n}^{\circ} 188 \mathrm{sq}$. 
et du mineur étranger, qui se voient reconnaître la capacité d'intenter des actions relatives à leur situation particulière, dans certaines limites, d'âge ou d'autorisation. Ainsi la capacité du mineur étranger à intenter une action relative à sa nationalité ne lui est reconnue qu'à compter de l'âge de 16 ans, et le mineur salarié ne peut agir lui-même devant le Conseil des prud'hommes qu'avec l'autorisation de ses parents ou de la juridiction ${ }^{26}$. Ces hypothèses de capacité sont fondées sur l'engagement contracté par le mineur: contrat de travail, mariage, ou / et sur le caractère personnel de l'acte en cause.

La capacité procédurale du mineur est également reconnue lorsque celui-ci par son comportement délictueux peut constituer un danger pour lui-même et pour la société. Le mineur délinquant est en effet partie et capable dans les procédures pénales diligentées contre lui, sous réserve d'être doué de discernement ${ }^{27}$. On peut se demander si l'incapacité persistante du mineur victime même discernant n'est pas incongrue face à la capacité du mineur délinquant... L'incapacité protectrice du mineur a-t-elle encore lieu d'être lorsque le danger s'est réalisé alors qu'elle est écartée lorsque la menace d'un danger fonde l'ouverture d'une procédure d'assistance éducative? Il paraît en outre difficile d'expliquer au mineur victime qu'il ne peut accéder au juge que par l'intermédiaire d'un représentant alors que le mineur délinquant a un accès direct au même juge.

L'avocat d'enfant, susceptible d'intervenir dans le cadre des actions dans lesquelles l'enfant est partie et capable, mais aussi lorsque ce dernier n'est pas capable, aux côtés de ses parents ou de son administrateur ad hoc, constitue un acteur central pour la participation de l'enfant aux procédures judiciaires. Depuis une dizaine d'années, il joue également un rôle important lorsque le mineur, seulement concerné en fait par la procédure, est entendu. L'avocat d'enfant peut en effet accompagner l'enfant lors de son audition et les avocats spécialisés ont donné à son rôle toute sa spécificité; il s'agit en effet d'accompagner sans représenter, d'aider l'enfant à exprimer son avis sans parler à sa place.

L'exercice par le mineur de son droit à participer aux procédures judiciaires, quelle que soit la forme qu'il revêt, est par ailleurs subordonné à l'information du mineur concerné par la procédure à propos des droits procéduraux dont il est titulaire ${ }^{28}$. La Convention européenne sur l'exercice des droits de l'enfant affirme, dans son préambule, que les enfants doivent recevoir des informations pertinentes afin que leurs droits et leurs intérêts supérieurs puissent être promus. L'importance de l'information de l'enfant témoigne d'une approche globale de la participation de l'enfant à la procédure et d'une volonté réelle de lui donner les moyens d'une participation effective. La Convention précise que l'autorité judiciaire doit s'assurer que l'enfant a reçu toute information pertinente. Il semble cependant que ce droit à l'information de l'enfant n'a pas encore véritablement reçu en droit interne de consécration formelle.

Il faut enfin noter que les différentes formes de participation du mineur aux procédures qui le concernent ne sont pas exclusives les unes des autres: l'enfant peut être entendu et représenté notamment lorsqu'il est victime d'une infraction pénale; il peut se voir reconnaître le statut de partie, et la capacité d'exercer des droits procéduraux, tout en étant entendu comme dans le cadre de la procédure d'assistance éducative lorsqu'il est doué de discernement; enfin, l'avocat et l'administrateur ad hoc peuvent conjuguer leurs efforts auprès du mineur victime ou objet d'une procédure d'assistance éducative.

Derrière cette esquisse des différentes formes de participation de l'enfant aux procédures judiciaires qui le concernent, apparaissent les contours d'un nouveau droit procédural de l'enfant: celui d'être associé au processus de décision qui le concerne. Droit intermédiaire entre la capacité et l'incapacité, ente l'autonomie et la soumission à l'autorité parentale, il permet de concilier la protection de l'enfant avec le respect de sa personne et de sa volonté. Il permet surtout d'associer l'enfant à la définition de son propre intérêt, critère majeur de la plupart des procédures concernant les enfants. Notion cadre, notion floue, formule magique, l'intérêt de l'enfant souffre certainement d'un déficit de définition, dont on sait qu'elle est impossible. Si on ne peut définir l'intérêt de l'enfant, a priori, sous peine d'ériger des dogmes toujours dangereux, on peut proposer des moyens de le déterminer et le droit de l'enfant d'être associé à sa définition en est un, essentiel.

26. Ibid., $\mathrm{n}^{\circ} 208 \mathrm{sq}$.

27. P. Bonfils, «Les juridictions répressives pour mineurs », Dr. fam., 2006, Études n ${ }^{\circ} 15$.

28. A. Gouttenoire, «Dessine-moi une procédure ou le statut procédural de l'enfant en Europe», in D. Gadbin et F. Kernaleguen, Le Statut juridique de l'enfant dans l'espace européen, Bruxelles, Bruylant, 2004, p. 319. 
